\title{
Prosthodontic Rehabilitation of Anterior Bony Defect with Fixed Removable Bridge System: - A Claspless Approach
}

Keywords: Andrew's bridge; Anterior ridge defects; Bar and clip assembly; Siebert's nomenclature

\begin{abstract}
Anterior Ridge defects as a result of trauma, congenital abnormalities of cleft palate require complex treatment procedures of hard and soft tissue augmentation prior to the prosthetic rehabilitation. In certain cases, where surgical intervention in the face of soft or hard tissue augmentation cannot be carried out, unconventional treatment options like fixed removable bridge system can be considered to provide esthetic and functional prosthesis. This case report aims at presenting a non-surgical as well as economical treatment alternative to restore and rehabilitate anterior bony defects using canines as abutments for fixed component followed by a removable component by a claspless approach.
\end{abstract}

\section{Introduction}

Resorption of alveolar bone following loss of teeth is an inevitable outcome. Also loss of hard tissue is magnified to have large vertical and horizontal component arising as a result of trauma, cleft palate defects (acquired or congenital) surgical ablation, and bone resorption following failure of grafting procedures. This leads to complexity of edentulous spaces that pose prosthodontic challenge, because it is not only difficult to replace missing teeth esthetically but also to achieve complete closure of underlying defect. Siebert identified three basic ridge deformities: buccolingual loss of tissues (Class I) apicocoronal loss of tissues (Class II) and combination of a buccolingual and apicocoronal loss of tissues (Class III) [1]. For quantitative assessment, a more detailed classification was proposed by Struder et al. that measured vertical and horizontal component of the defect [2]. To rehabilitate missing hard and soft tissues in cases of ridge defects particularly in esthetic zone requires meticulous and skillfull efforts [2]. Such defects can be treated in number of ways; one of the non-surgical treatment alternatives was introduced by Dr. James Andrews (Louisiana), as fixed removable Andrews Bridge System (Institute of Cosmetic Dentistry, Amite, La). This system is best indicated for cleft palate patients with congenital or acquired defects; its design meets the requirements of esthetics, comfort, phonetics, and hygiene maintenance along with favorable stress distribution between the abutments and residual soft tissues. This assembly is a combination of fixed and removable components; fixed component consists of two retainers cemented over abutment teeth that are joined by a rectangular bar that spans over the curvature of residual ridge. This bar serves to provide engagement to resilient

\section{Eournal of Oral Biology}

\author{
Priya Vaswani*, Pronob Sanyal, Abhijeet Kore and \\ Ankur Prajapati
}

Department of Prosthodontics, Crown and Bridge, School of Dental Sciences, Krishna Institute of Dental Sciences Deemed University, Karad, Maharastra, India

\section{*Address for Correspondence}

Priya Vaswani, Department of Prosthodontics, Crown and Bridge, School of Dental Sciences, Krishna Institute of Dental Sciences, Deemed University, Karad-415110, Maharastra, India, Tel: 09833014506/0974266949; E-mail: vaswani.priya577@gmail.com

\section{Submission: $29 \mathrm{Mar}, 2016$}

Accepted: 15 April, 2016

Published: 20 April, 2016

Copyright: (๑) 2016 Pronob SK. This is an open access article distributed under the Creative Commons Attribution License, which permits unrestricted use, distribution, and reproduction in any medium, provided the original work is properly cited.

Reviewed \& Approved by: Dr. Rajiv Saini, Department of Periodontology \& Oral Implantology, Pravara Institute of Medical Sciences, Maharastra, India

plastic retentive clips or sleeves embodied within a removable component which replaces missing teeth within the bar area [3]. The vertical walls of the bar and frictional fit of the sleeve provides much needed retention and stability for Andrews's bridge system. This type of prosthesis has qualities of both the fixed partial and removable partial denture [4].

\section{Case Report}

A 21 year old young female patient reported to Department of Prosthodontics, School of Dental Sciences, KIMSDU, Karad, India with a chief complaint of missing upper front teeth, her past dental history revealed repair of cleft lip and palate done in her childhood, also a closure of oronasal communication with grafting using an alloplastic bone graft (Nova Bone) to augment an alveolar cleft located in right anterior maxillary region was attempted. Her medical history did not present any contraindication to dental treatment. Intraoral examination revealed, closure of oronasal communication, an alveolar cleft extended from labial to palatal cortical plate suggesting resorption of alveolar graft, Grade III mobility with respect to 21, 22 and congenitally missing 11 and 12 .With a surgical consent, Grade III mobile incisors were extracted. Since the patient did not give consent

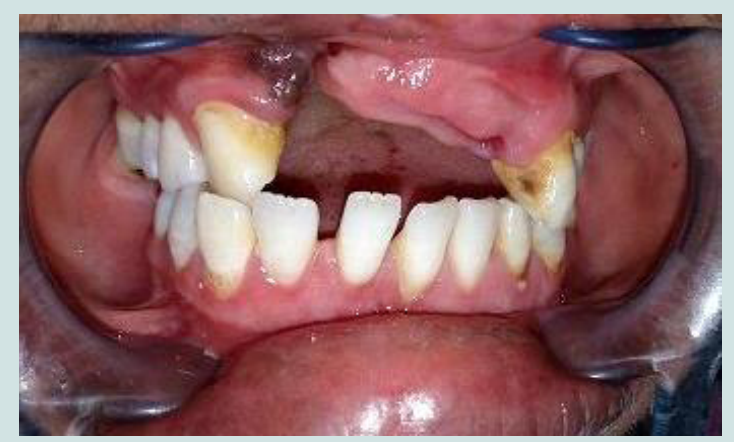

Figure 1: Intra-oral view of cleft segment, post extraction of mobile incisors. 
Citation: vaswani P, sanyal P, kore A, prajapati A. Prosthodontic Rehabilitation of Anterior Bony Defect with Fixed Removable Bridge System: - A Claspless Approach. J Oral Bio. 2016; 3(1): 4.

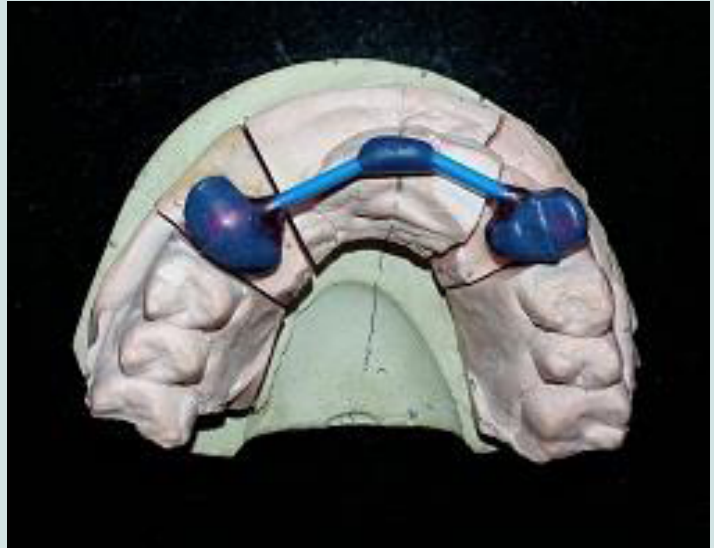

Figure 2: Castable resin Bar attached in two segments along a common vertical plane.

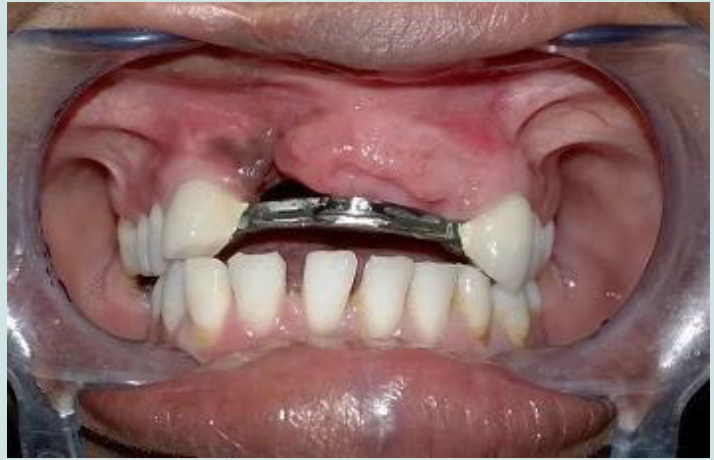

Figure 3: Cemented Rectangular bar with ceramic facing crowns

for repeat surgical soft tissue augmentation or grafting to restore the defect by placement of dental implants this treatment modality was ruled out. On contrary, the defect being large enough to be selfcleansable, a fixed partial denture was discarded as treatment option. Anon-surgical treatment that is Andrews's bridge system was opted for replacement of missing teeth as well as to restore the adjoining defect. Post extraction, clinical situation revealed that the defect was cone shaped, depending upon tissue loss it was categorized as Siebert's class III. According to semi-quantitative analysis, the vertical and horizontal component of defect measured greater than $6 \mathrm{~mm} \times 6$ $\mathrm{mm}$ indicating severe alveolar ridge defect (Figure1).

Intentional Root canal therapy was performed with the abutment teeth 23 and13, as 13 was cross bite in relation to opposing dentition. These teeth were prepared to receive ceramic facing crowns, a final impression was made in putty wash consistency of silicone impression material (Aquasil, Denstplytm India), and impression was poured in Type IV gypsum (Kalrock Kalabhai Karson, Mumbai Pvt Ltd). Removable die of abutments were prepared. With facebow transfer, the cast was mounted, a wax mock -up provided visual appeal of the existing smile. Wax pattern for coping was fabricated in type II inlay wax (DFS: Germany), prefabricated rectangular bar in castable resin (Standard length: Bredent UK) having a central groove on either side was then fixed onto mesial aspect of wax pattern of abutment teeth ,the bar was attached in two segments such that it followed the residual ridge curvature, care was taken so that the occlusal portion of both the segments were in same plane to facilitate common path of withdrawal and placement of removable component (Figure 2).

The height of the bar was kept to $2 \mathrm{~mm}$ and adequate amount of clearance from the gingiva was maintained during the wax up. The bar framework was invested, burned-out, and casted according to the manufacturer's instructions. The bar along with the copings was casted in cobalt chromium alloy (Wiron 99 BEGO, USA), metal try-in was performed so as to check for fitting of coping onto their abutments, along with occlusal clearance of the rectangular bar in relation to opposing dentition. In case where the bar encroaches onto underlying soft tissue excessive proliferation of tissues can occur due to irritation, relief by trimming should be provided so as to have an adequate clearance of 1.5 to $2 \mathrm{~mm}$. After satisfactory shade selection and ceramic layering, bar was finished and polished overall except for the groove present in the center of the bar so as to preserve frictional gripping of retentive sleeves. Entire fixed component was cemented over the abutment teeth using glass ionomer cement (Fuji 1, GC, USA) (Figure 3)

An irreversible hydrocolloid impression (Alginate-Marieflex, Septodent) was made to obtain a cast for fabrication of removable component. The parameters of shade, size and shape of prosthetic teeth (Ivoclar Vivadent India) were selected depending upon the facial features of the patient, age and gender. Since there was adequate

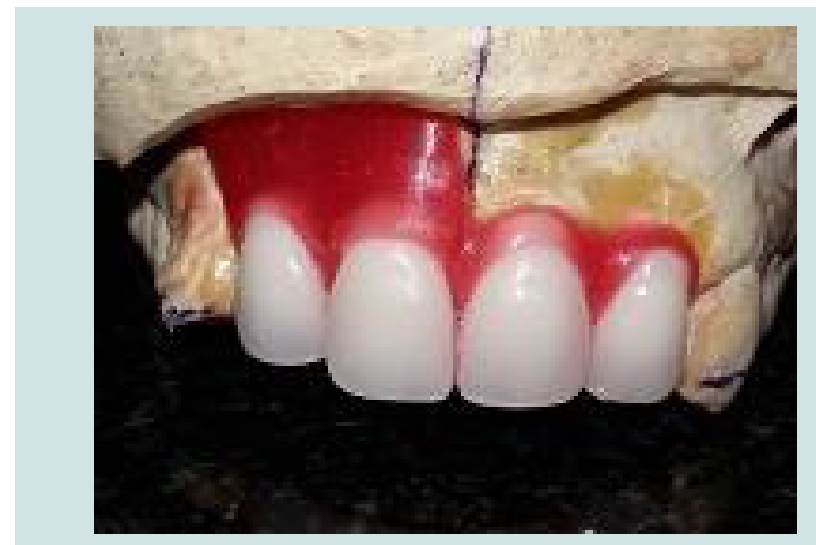

Figure 4: Wax-up of Removable component.

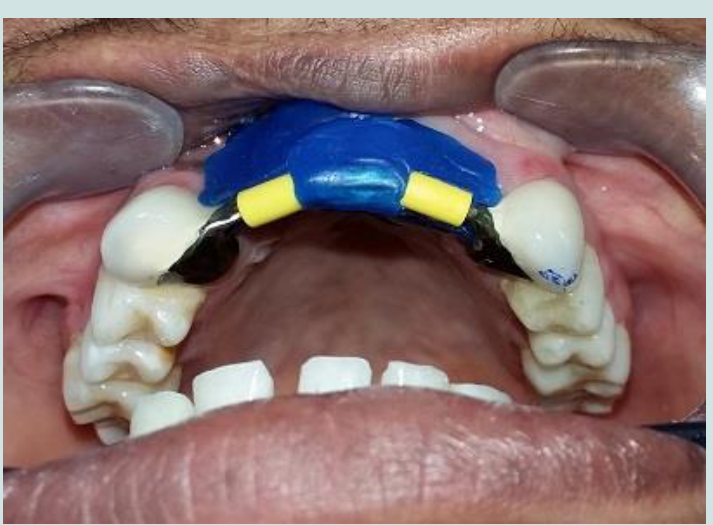

Figure 5: Blocking of Undercut with retentive clips attached. 

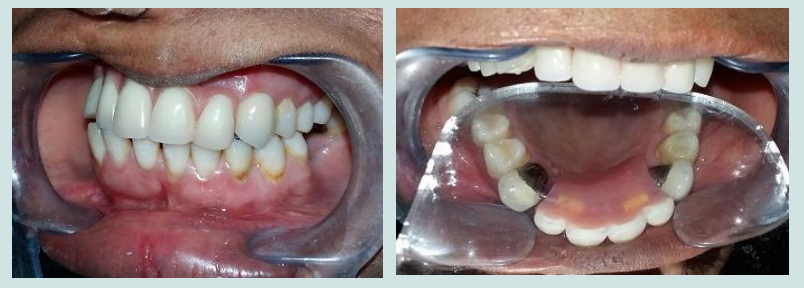

Figure 6: Superstructure prosthesis with resilient clips.

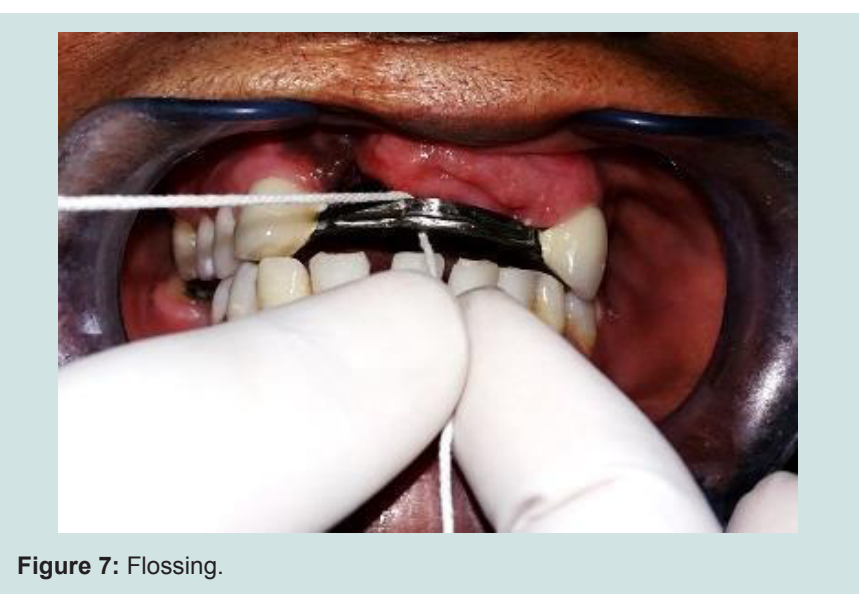

labial cortical bone in upper left anterior region, gingival flange was fabricated only on the right side so as to aid in closure of defect with reduced denture bulk (Figure 4).

After satisfactory esthetic trial of temporary record base carrying prosthetic teeth, it was processed into heat cure acrylic resin (DPI Heat cure denture base Mumbai India). Retentive clips (Normal medium friction: Bredent UK) were attached over the rectangular bar. Potential undercuts intraorally were blocked (Figure 5), space was created in the intaglio surface for autopolymersisng resin, passive fit of the removable component over the bar was checked. Small quantity of fluid consistency of autopolymersisng resin (DPI Cold cure Acrylic Repair Material: Mumbai India) was loaded onto the intaglio surface of the denture and placed over the bar such that the retentive clips were picked up in the removable component.

The patient was trained for placement and removal of the denture. Hygiene instructions included daily interdental brushing (single tuft brushes) and flossing (Figure 7) beneath the rectangular bar and adjacent abutment teeth. (Figure 6).

Periodic recall at first week, and first month followed by 3 months upto an year were carried out with no loss of retention or adaptability with either of components of final prosthesis (Figure 8)

\section{Discussion}

This article reports prosthetic rehabilitation of a unilateral cleft lip and palate in a 21 year old female patient who had congenitally missing anterior teeth with a clasp less approach. In this case, a surgical repair of oronasal communication was attempted with grafting alloplastic bone, post-operative shrinkage of tissue lead to large alveolar defect. Congenitally missing anterior teeth are common in cleft palate patients of which frequently missing are lateral incisors, although canine and central incisors can also be affected with remaining teeth being malformed or malposed. Only $9 \%$ of the patients with the anterior teeth missing between the two canines did not have ridge defects [5]. This situation is complicated by dysphagia, hypernasal speech, oronasal regurgitation and unaesthetic appearance. Bone support adjacent to the cleft is generally compromised. Hence complete obturation of defect to aid in swallowing, speech with easy maintainability is deemed necessary. Quoting the famous Devan's dictum "Our objective should be perpetual preservation of what remains, than meticulous reconstruction of what is lost" [6], Hygiene maintenance of tissues supporting and surrounding the prosthesis plays a crucial role for its long term success. For most advantage of a combination prosthesis is that it enhances the patient's ease and comfort for maintenance of soft tissues particularly in defect prone areas. In the above case, conventional anterior fixed partial denture would have displayed unacceptably long pontics with large, empty interproximal embrasures due to increased mesiodistal space, large black triangles and ulceration due to interdental flossing owing to less self -cleansable edentulous span, this outcome is highly undesirable for a young female patient. A conventional cast partial denture would have been unaesthetic due to the anterior clasp and a palatal major connector that would otherwise lead to anterior posterior torquing movement to a magnitude similar that seen in Class 1 Kennedy's situation. Implant placement was a dubious alternative as the loss of labial cortical plate would have made the implant placement procedure difficult. Even with the bone grafting in such cases the success of osseointegration is questionable [7]. Hence, within the limitations of case presented, Andrews's bridge was thought to be the most acceptable economic solution for the patient, due to her financial constraints.

The principle advantage is flexibility in placing denture teeth, restores the facial contour, thereby enhancing esthetics $[8,9]$ has superior adaptability, comfort, and improves phonetics $[7,10]$. Occlusal forces are directed towards the long axis of abutment teeth. The seating and close adaptation of acrylic saddle on the ridge absorbs all the forces, curved bar ensures lifting and depressing forces are shared by all sectors, the system acts as a stress breaker while transmitting unwanted leverage forces. There is no plate as in removable partial

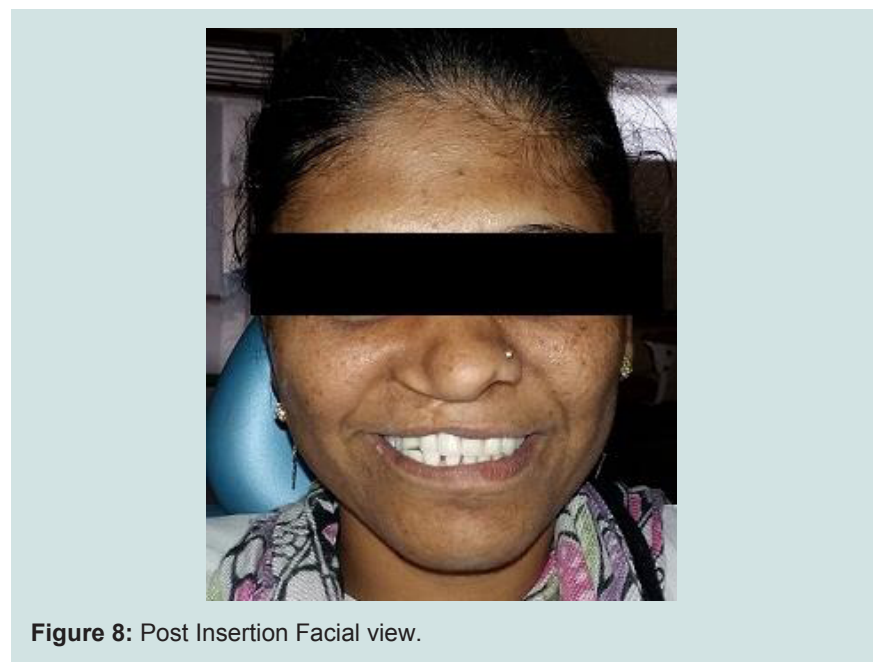


Citation: vaswani $\mathrm{P}$, sanyal $\mathrm{P}$, kore A, prajapati A. Prosthodontic Rehabilitation of Anterior Bony Defect with Fixed Removable Bridge System: - A Claspless Approach. J Oral Bio. 2016; 3(1): 4.

denture, and prosthetic assembly can be relined if the ridge resorbs [11]. The main drawback in this system is the removable component, which reduces the acceptance towards the treatment, especially a younger individual. However, since the replacement was limited to the aesthetic zone a claspless removable denture was planned and inserted .With proper counseling and reassurance, the patient can be made to accept the treatment and also be motivated for proper maintenance of the prosthesis. The retentive clips have the tendency to wear, resulting in loss of retention. Therefore, regular checkup and chair side replacements of retentive clips are required. In this case report, with proper analysis of the diagnostic factors and involvement of the patient in the decision-making process, fixed removable prosthesis need not limit itself to be just a prosthetic alternative but a definite treatment option.

\section{Conclusion}

Partially edentulous spaces with accompanying ridge defects that cannot be augmented surgically are best treated economically with Andrew's bridge. This treatment modality not only replaces the missing teeth but achieves complete closure of the defect, more importantly it aids in hygiene maintenance, with positive impact on psychologic well being, generates confidence along with positive attitude towards life particularly in patients who are trauma inflicted and those who exhibit congenital or acquired clefts.

\section{References}

1. Seibert JS (1983) Reconstruction of deformed, partially edentulous ridges, using full thickness onlay grafts. Part I. Technique and wound healing. Compend Contin Educ Dent 4: 437-453.

2. Studer S, Naef R, Scharer P (1997) Adjustment of localized alveolar ridge defects by soft tissue transplantation to improve mucogingival esthetics: a proposal for clinical classification and evaluation of procedures. Quintessence Int 28: 785-805.

3. Everhart RJ, Cavazos E Jr (1983) Evaluation of a fixed removable partial denture: Andrews Bridge System. J Prosthet Dent 50: 180-184.

4. Mueninghoff LA, Johnson MH (1982) Fixed-removable partial denture. J Prosthet Dent 48: 547-550.

5. Abrams H, Kopczyk RA, Kaplan AL (1987) Incidence of anterior ridge deformities in partially edentulous patients. J Prosthet Dent 57: 191-194.

6. DeVan MM (1952) Basic principles of impression making. J Prosthet Dent 2: $26-35$.

7. Ravi Shankar Y, Raju AV, Srinivasaraju D, Babu JP, Kumar DR, et al. (2011) A fixed removable partial denture treatment for severe ridge defect. Int J Dent Case Reports 1: 112-118.

8. Malament KA, Neeser S (2004) Prosthodontic management of ridge deficiencies. Dent Clin North Am 48: 735-744, vii.

9. Kaurani P, Rupandeep, Samra K, Kaurani M, Padiyar N (2013) Prosthodontic Rehabilitation of a case with an anterior ridge defect using andrews bridge. Indian J Dent Sci 2: 100-103.

10. Vaswani P, Sanyal P, Prajapati A (2015) Comparison of speech articulation and intelligibility in palatally countered dentures using a novel rugae duplication technique: A Clinical Study. Int J Dent Res 3:15-20.

11. Sanyal P, Badwaik P (2011) Methods of identification of complete dentures. People's J Sci Res 4: 61-64. 